\title{
I see what you mean: Theta power increases are involved in the retrieval of lexical semantic information
}

\author{
Marcel C.M. Bastiaansen ${ }^{\mathrm{a}, \mathrm{b}, *}$, Robert Oostenveld ${ }^{\mathrm{a}, \mathrm{c}}$, Ole Jensen ${ }^{\mathrm{a}}$, Peter Hagoort ${ }^{\mathrm{a}, \mathrm{b}}$ \\ ${ }^{a}$ Max Planck Instituut for Psycholinguistics, Nijmegen, The Netherlands \\ ${ }^{\mathrm{b}}$ F.C. Donders Centre for Cognitive Neuroimaging, Radboud University Nijmegen, P.O. Box 9101, 6500 HB Nijmegen, The Netherlands \\ ${ }^{\mathrm{c}}$ Center for Sensori-Motor Interaction, Aalborg University, Denmark
}

Accepted 26 October 2007

Available online 8 February 2008

\begin{abstract}
An influential hypothesis regarding the neural basis of the mental lexicon is that semantic representations are neurally implemented as distributed networks carrying sensory, motor and/or more abstract functional information. This work investigates whether the semantic properties of words partly determine the topography of such networks.

Subjects performed a visual lexical decision task while their EEG was recorded. We compared the EEG responses to nouns with either visual semantic properties (VIS, referring to colors and shapes) or with auditory semantic properties (AUD, referring to sounds).

A time-frequency analysis of the EEG revealed power increases in the theta $(4-7 \mathrm{~Hz})$ and lower-beta $(13-18 \mathrm{~Hz})$ frequency bands, and an early power increase and subsequent decrease for the alpha $(8-12 \mathrm{~Hz})$ band. In the theta band we observed a double dissociation: temporal electrodes showed larger theta power increases in the AUD condition, while occipital leads showed larger theta responses in the VIS condition.

The results support the notion that semantic representations are stored in functional networks with a topography that reflects the semantic properties of the stored items, and provide further evidence that oscillatory brain dynamics in the theta frequency range are functionally related to the retrieval of lexical semantic information.
\end{abstract}

(C) 2008 Published by Elsevier Inc.

Keywords: EEG; Language comprehension; Oscillatory brain dynamics; Theta rhythm; Semantic representations; Psycholinguistics; Time-frequency analysis; Memory retrieval

\section{Introduction}

It has been proposed repeatedly that semantic representations of words, and objects, are neurally implemented in distributed networks carrying sensory, motor and/or more abstract functional information (e.g., Damasio, 1990; Farah \& McClelland, 1991; Warrington \& McCarthy, 1987; Warrington \& Shallice, 1984). A more specific hypothesis is that the exact topology of such networks is

\footnotetext{
* Corresponding author. Address: F.C. Donders Centre for Cognitive Neuroimaging, Radboud University Nijmegen, P.O. Box 9101, 6500 HB Nijmegen, The Netherlands. Fax: +31 243610989.

E-mail address: marcel.bastiaansen@fcdonders.kun.nl (M.C.M. Bastiaansen).
}

at least partly determined by the semantic properties of the word or object concept (Martin \& Chao, 2001; Pulvermueller, 1999, 2001). This contrasts with the view put forward by others (e.g., Devlin et al., 2002; Tyler \& Moss, 2001; Tyler, Moss, Durrant-Peatfield, \& Levy, 2000) that semantic representations are stored in a distributed network in which there is no neural specificity as a function of semantic property.

According to the first view, manipulable objects such as tools are strongly linked to motor behavior, and therefore their representational networks should comprise a significant amount of neurons in motor cortex. Animals, which are most of the time (visually) perceived rather than manipulated, should be represented by networks that partly reside in visual cortex. Given anatomically confined 
lesions/damage to neural tissue, the topographical distinction between the representations of both categories is likely to lead to selective damage to the representations in one category. Support for this view comes from a large number of neuropsychological studies (see Forde \& Humphreys, 1999; Saffran \& Sholl, 1999 for reviews), that have shown the existence of category-specific semantic impairments in patients, for instance a relatively selective impairment for naming/recognizing animals as opposed to tools, or-less frequently-vice versa. These findings are at least compatible with the view that the semantic properties of words or objects partly determine the topography of their neural representation (although they can be interpreted in different ways, e.g., Tyler \& Moss, 2001).

More direct support comes from a number of hemodynamic neuroimaging studies. In a PET experiment, Martin et al. (Martin, Haxby, Lalonde, Wiggs, \& Ungerleider, 1995) found that generation of color words selectively activated a region in the ventral temporal lobe just anterior to the area involved in the perception of color, whereas generation of action words activated a region in the middle temporal gyrus just anterior to the area involved in the perception of motion. In another PET study (Martin, Wiggs, Ungerleider, \& Haxby, 1996), these authors showed that naming pictures of tools activated premotor areas, while naming pictures of animals activated medial occipital areas. Within the language domain, a recent fMRI study (Hauk, Johnsrude, \& Pulvermueller, 2004) showed that the visual presentation of verbs related to the execution of face, arm and leg movements (e.g., to lick, to pick and to kick) differentially activated areas in the motor cortex that were adjacent to or overlapping with the areas that were activated during actual movements of the corresponding body parts. Similarly, recent fMRI data (Vitali et al., 2005) show different effective connectivity patterns for viewing tools vs. animals, with tools engaging areas in frontal and premotor areas, and animals engaging visual association areas. It should be noted however that other studies failed to find such semantically specific activations during semantic processing (e.g., Noppeney \& Price, 2002).

Together, the available data largely support the view that spatially distributed functional networks form the basis of semantic representations, and that the topographies of these networks correspond to the semantic properties of individual items. However, in order to answer the question of how such functional networks are formed, one has to gain insight into the temporal dynamics of their activation. Here, we present EEG data showing that oscillatory neuronal dynamics in the theta frequency range are involved in the activation of functional networks with semantically specific topography.

The temporal dynamics of neuronal activity can be investigated with neuroimaging methods that yield high temporal resolution such as EEG or MEG. While some studies have investigated the scalp topography of eventrelated potentials (ERPs) elicited by the presentation of different categories of words (Hauk \& Pulvermueller, 2004;
Pulvermueller, Harle, \& Hummel, 2001), the oscillatory dynamics of EEG and MEG data presumably provide another window on the dynamic formation of functional networks. The reason for this is that an (indirect) link can be made between changes in neuronal synchrony on the one hand, and changes in the oscillatory activity that is present in scalp-recorded EEG and MEG on the other hand. The amplitude (or power) of oscillatory activity is indicative of synchronization changes because the synchronization of local groups of neurons, the activities of which are picked up by one and the same sensor, will result in larger EEG/MEG amplitudes for that sensor. It follows that local changes in synchrony of oscillatory firing patterns lead to changes in amplitude (or power) of rhythmic EEG/MEG activity at the single-trial level. The phase of EEG/MEG oscillations is important because synchronization of oscillatory firing patterns between spatially distant neuronal populations lead to increases in coherence, or in phase synchronization, between two (or more) concurrently measured EEG or MEG signals (Bastiaansen \& Hagoort, 2003; Singer, 1999; Varela, Lachaux, Rodriguez, \& Martinerie, 2001, see Pulvermueller, 1999 for arguments of how this mechanism applies to the representation of lexical semantics).

In sum, a topographical analysis of event-related changes of either power or coherence in oscillatory EEG or MEG activity during language processing tasks would be informative in establishing whether synchronous functional networks with distinct scalp topographies constitute the representation of different categories of words. A few studies have provided data that speak to this issue. In a number of studies aimed at characterizing EEG coherence changes elicited by different word categories, Weiss and colleagues (see Weiss \& Mueller, 2003 for review) found different coherence patterns in the lowerbeta frequency range (roughly $13-18 \mathrm{~Hz}$ ) between concrete and abstract nouns, and between concrete nouns and verbs. However, the authors did not attempt to relate coherence topography to semantic properties in these studies. A study by Pulvermueller (Pulvermueller, Lutzenberger, \& Preissl, 1999) did attempt to relate semantic properties to the scalp topography of EEG power changes. In a lexical decision task, nouns were contrasted with verbs, assuming that nouns are perception-related and should therefore have a relatively larger neuronal representations in occipital cortex, while verbs are action-related and should have representational network elements in motor cortex. The authors found power decreases in the gamma frequency range compared to baseline, that were smaller for verbs than for nouns at central electrodes, but smaller for nouns than for verbs over occipital electrodes. These data were interpreted to reflect different network topographies that followed the semantic properties of the stimuli.

Other studies have related semantic memory operations to power changes in the alpha frequency band (for review, see Klimesch, 1999). It should be noted however, that in 
most of these studies, the term semantic memory is used in the sense of declarative (as opposed to episodic) memory. In language comprehension theories however, the term semantic is used in a more narrow sense, referring to the meaning aspect of words (as opposed to syntax, phonology etc.). In the remainder of this paper, we will use the term semantic to refer to the more narrow, psycholinguistically defined process of attributing meaning to linguistic material. However, some studies do address semantic processing in this more narrow sense (e.g., Klimesch, Doppelmayr, Pachinger, \& Ripper, 1997a; Klimesch, Doppelmayr, Pachinger, \& Russegger, 1997b; Rohm, Klimesch, Haider, \& Doppelmayr, 2001). Generally, the results of these studies support the notion that semantic processing is indeed related to power decreases in the upper alpha band (roughly $10-12 \mathrm{~Hz}$ ).

We further pursue the issue of whether words with different semantic properties are neurally represented in topographically distinct functional networks, by taking a different approach in constructing stimulus material. Rather than relying on a correlation between semantic features and specific word classes, we constructed two sets of nouns, one referring exclusively to colors and shapes, and the other referring exclusively to sounds. Thus, one set of stimuli only contained nouns with visual semantic properties, while the other set only contained nouns with auditory semantic properties. While their EEG was measured, subjects performed a visual lexical decision task, in which we contrasted the 'visual' nouns with the 'auditory' nouns (note that both were presented to the subjects in the visual modality). Given our hypothesis, the former set of nouns should activate a network with a relatively large component in the visual cortex, whereas the latter set should lead to the emergence of a network that extends into auditory cortex. Because event-related changes in power reflect changes in local synchrony, while eventrelated changes in phase coherence between different sensors reflect changes in long-range synchrony (Bastiaansen \& Hagoort, 2003; Varela et al., 2001) we expected these local differences in network topography to be expressed as differential power increases over auditory and visual areas. More specifically, we expected EEG power to be larger over the auditory cortex following words with auditory semantic properties compared to words with visual semantic properties, while the opposite pattern was expected over the visual cortex. On the basis of the existing literature on oscillatory EEG/MEG dynamics during language processing, we hypothesized this differential power increase to be manifest in one (or more) of the following frequency bands: theta $(4-7 \mathrm{~Hz}$; based on Bastiaansen, van Berkum, \& Hagoort, 2002b; Bastiaansen, van Berkum, \& Hagoort, 2002c; Hagoort, Hald, Bastiaansen, \& Petersson, 2004), alpha $(8-12 \mathrm{~Hz}$; based on the studies reviewed in Klimesch, 1999), lower-beta (13-18 Hz; based on the studies reviewed in Weiss \& Mueller, 2003) or gamma (above $30 \mathrm{~Hz}$; based on Hagoort et al., 2004; Pulvermueller et al., 1996, 1999).

\section{Materials and methods}

\subsection{Subjects}

Sixteen students (4 male) from the Radboud University Nijmegen (aged 21-36 years), were paid for their participation after having given informed consent in writing, according to the Declaration of Helsinki. All were native Dutch speakers with normal or corrected-to-normal vision. They were right-handed, as assessed by a self-report. None of the participants had any neurological impairment, neurological trauma, and none used neuroleptics.

\subsection{Stimulus material}

The item set that is critical for the present study consisted of two sets of 59 nouns each. One set referred to colors and shapes (i.e., a set with visual semantic properties, VIS). Examples of these stimuli are (the Dutch equivalents of) 'red', 'purple', 'square', 'curve'. The other set referred to sounds (i.e., with auditory semantic properties, AUD). Examples are the Dutch equivalents of 'whistle', 'scream', 'sigh', 'echo'). Both sets were carefully matched using the CELEX lexical database (Baayen, Piepenbrock, \& van Rijn, 1993) for word length (between 5 and 8 characters, mean length of 6.5 characters for both sets) and word frequency (between 0 and $1.5 \log$ frequency of occurrence per million, mean of $0.648 \mathrm{log}$ frequency for both sets). Other stimuli were also included in the experiment, but they are of no interest for the present purpose and can be considered to be filler items. They consisted of 156 correct Dutch words (other than the 120 AUD and VIS items described above), 137 pseudowords, 137 consonant strings, 78 pictures of tools and animals, and 78 scrambled pictures. Thus a total of 704 stimuli were used.

\subsection{Design and procedure}

The stimuli detailed above were presented in a delayed two-choice reaction time task. For the orthographic stimuli (words, pseudowords and consonant strings), the subject's task was to indicate, by pressing one of the response buttons, whether or not the orthographic string constituted a legal Dutch word (i.e., lexical decision task).

Subjects were seated in a comfortable armchair located in a dimly illuminated, sound-attenuating, and electrically shielded room. Stimuli (the orthographic stimuli were presented in font size 36 as white letters on a black background) were presented on a monitor located approximately $1.2 \mathrm{~m}$ in front of the subject. Responses were given by pressing one of two response buttons, mounted on the left and right arms of the chair. One button was labeled 'existing', the other was labeled 'not existing'. The mapping of labels onto response sides was counterbalanced over subjects.

Each trial started with a $1000 \mathrm{~ms}$ baseline interval, in which no stimuli were presented. Then the stimulus was 
presented for $1000 \mathrm{~ms}$, after which it was replaced by a fixation cross. The fixation cross stayed on for a randomly variable interval of between 2000 and $4000 \mathrm{~ms}$ (in steps of $1000 \mathrm{~ms}$ ), and indicated that the subjects had to give a response (i.e., a delayed response), and were allowed to blink. The disappearing of the fixation cross indicated the start of the following trial. A delayed response was used in order to minimize the overlap of pre-movement spectral power changes that are known to occur in the EEG (Pfurtscheller \& Berghold, 1989) with the hypothesized post-stimulus power changes. On average, each trial was $5000 \mathrm{~ms}$ long.

Trials were presented in four blocks of 176 trials each. Eight different versions of the experiment were created by randomly assigning stimuli to one of the blocks, in order to eliminate order effects. Each version was used for two different subjects. Each block took approximately $14 \mathrm{~min}$ to complete, after which the subject was given a short break. Subjects were familiarized with the task in an initial training block consisting of 20 trials, that were not ana- lyzed. The complete experiment took approximately two hours to complete, including approximately $45 \mathrm{~min}$ of subject preparation time.

\subsection{Data recording and preprocessing}

The EEG was recorded from $62 \mathrm{Ag}-\mathrm{AgCl}$ ring electrodes mounted in an elastic cap (Easycap, Germany), each referenced to the left mastoid. The electrodes were placed in an equidistant montage (see e.g., the topographical map in Fig. 1 for a radial projection of the locations), with one electrode overlaying the right mastoid. Eye movements were monitored via a supra- to sub-orbital bipolar montage. The EEG and EOG signals were amplified with BrainAmp amplifiers (Brain Products, Germany), using a high-frequency cut-off of $70 \mathrm{~Hz}$ and a time constant of $10 \mathrm{~s}$. Electrode impedance was kept below $3 \mathrm{k} \Omega$ for the EEG recording and below $5 \mathrm{k} \Omega$ for the EOG recording. The EEG and EOG signals were digitized on-line with a sampling frequency of $500 \mathrm{~Hz}$, and stored for off-line analysis.

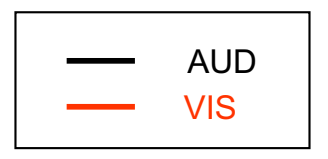

ERP time courses
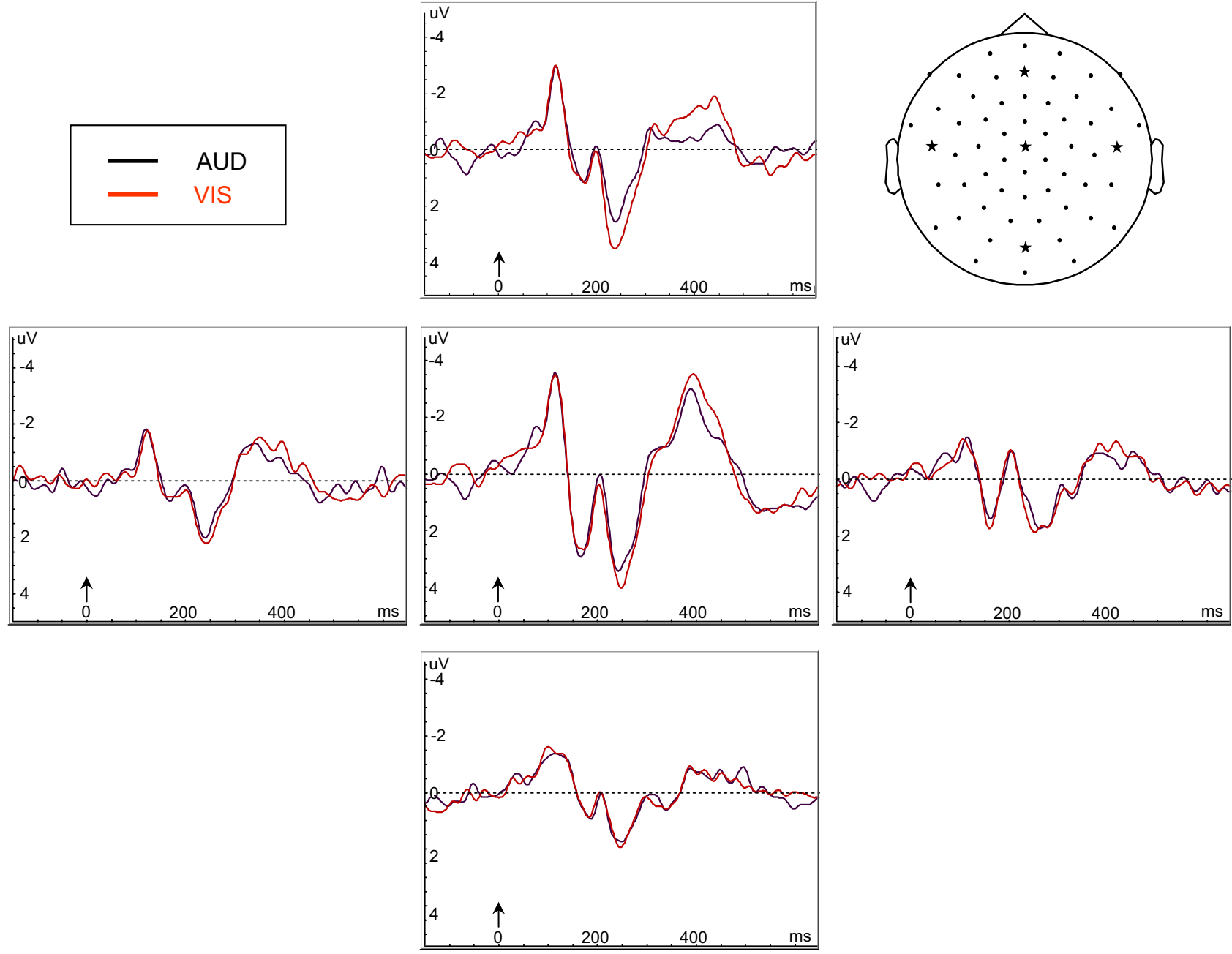

Fig. 1. Time course of grand average $(N=16)$ ERPs elicited by AUD and by VIS words, at representative channels. Channel positions relative to the subject's head are indicated by asterisks in the head plot in the top right-hand corner. 
The EEG data were then re-referenced to the algebraic average of both mastoids, and screened (through an automatic detection procedure and subsequent visual inspection) for eye movements, muscle artifacts, electrode drifting, and amplifier blocking in a critical window that ranged from $500 \mathrm{~ms}$ before to $1000 \mathrm{~ms}$ after stimulus onset. Trials containing such artifacts, and trials containing behavioral errors, were excluded from further analysis ( $12 \%$ on average).

\subsection{ERP analysis}

For the ERP analysis, the single-trial EEG data were baseline-corrected in an interval from $150 \mathrm{~ms}$ to $0 \mathrm{~ms}$ prestimulus, and averaged separately for the AUD and VIS conditions. Finally the data were band-pass filtered between 1 and $30 \mathrm{~Hz}$ to mimic conventional ERP filter settings.

\subsection{Time-frequency analysis}

A time-frequency representation (TFR) of the singletrial EEG data was constructed using a multitaper approach (Mitra \& Pesaran, 1999). Power was analyzed between 1 and $60 \mathrm{~Hz}$, in $1-\mathrm{Hz}$ steps, as a function of time and frequency using a sliding window technique. Window lengths were adapted to the different frequencies so as to contain five cycles. This yields a time/frequency resolution trade-off similar to that obtained with Morlet wavelets with a five-cycle width (c.f. Tallon-Baudry, Bertrand, Delpuech, \& Pernier, 1996). Multitapers were chosen to concentrate spectral energy over frequency bands that widened with increasing frequency, such that for a given frequency, concentration was over a band of $\pm 1 / 5$ of that frequency.

The TFRs of the single-trial data were averaged separately for the AUD and VIS conditions. The resulting average power values were then expressed as the percentage power increase or decrease relative to the power in a $500 \mathrm{~ms}$ prestimulus baseline interval. The resulting measure is similar to the Event-Related Desynchronization/Synchronization (ERD/ERS) measure developed by Pfurtscheller and colleagues (Pfurtscheller \& Aranibar, 1977, 1979 see Pfurtscheller \& Lopes da Silva, 1999 for a large collection of studies using this measure). In this terminology ERS stands for power increases, and ERD indicates a power decrease.

\subsection{Time-frequency analysis of the ERP data}

ERP components such as the N1-P1 complex and the N400 have the majority of their power concentrated in the delta, theta and alpha frequency bands (see, e.g., Makeig et al., 2002; Sakowitz, Quian Quiroga, Schurmann, \& Basar, 2005). Therefore it is conceivable that the results of the single-trial analyses, intended to focus on oscillatory (i.e., non-phase-locked) components, are partially brought about by differences in spectral content of the time-locked averages - i.e. the ERP data. In order to verify this, we applied the same multitaper analysis as we used for the time-frequency analysis of oscillatory components to the ERPs.

For a statistical analysis of the ERP power changes, we analysed the data in a $100-400 \mathrm{~ms}$ window after word onset (i.e., the time interval used for the analysis of the theta oscillations), and performed separate ANOVA's (see below) for the theta band $(4-7 \mathrm{~Hz})$ and the alpha band $(8-12 \mathrm{~Hz})$.

\subsection{Statistical analysis}

For a statistical analysis of both the ERP and TFR data, we constructed four regions of interest (ROIs) on an a-priori basis: a left- and a right-occipital region ( $\mathrm{LO}$ and $\mathrm{RO}$ ), and a left- and a right-temporal region (LT and RT). The reason we selected those ROIs was that, although we are aware that there is no one-to-one correspondence between scalp recordings and underlying neuronal generators, these scalp areas most closely correspond to the underlying visual and auditory cortices, respectively. The black ellipses in Fig. 2a indicate the location of these ROIs, and the electrodes they include. For the ERP data, the mean potentials in a $300-500 \mathrm{~ms}$ (the classical N400 time interval) poststimulus time window were averaged over the electrodes belonging to one and the same ROI. For the TFR data, the average power changes at each electrode were computed in four specific time-frequency windows (see results section), and the resulting values were averaged for the electrodes belonging to one and the same ROI. Note that we performed the ERP analyses in the classical N400 time window, because it is clear from electrophysiological psycholinguistics that the lexical effects that we were looking for are to be expected within this time window. For the TFR data however, much less is known about their antecedent conditions, so we analysed each of the oscillatory components in the time window where the observed effects were maximal. Both the ERP and the TFR data thus quantified were entered into ANOVA's for repeated measures with the factors Condition (AUD, VIS), Area (temporal, occipital) and Hemisphere (left, right). Significant interactions were clarified by breaking them down into simple effects. Because our hypotheses include the direction of the expected effects, we report one-tailed $p$-values.

\section{Results}

\subsection{ERP data}

Fig. 1 shows the time-course of the ERPs elicited by the AUD and VIS words at representative channels.

A negative component is observed, with a (grand-average) peak at $400 \mathrm{~ms}$ after word onset. We identify this component as the classical N400. This is confirmed by the postcentral topographical distribution (Fig. 2a). A slight difference in scalp topography appears to be present 

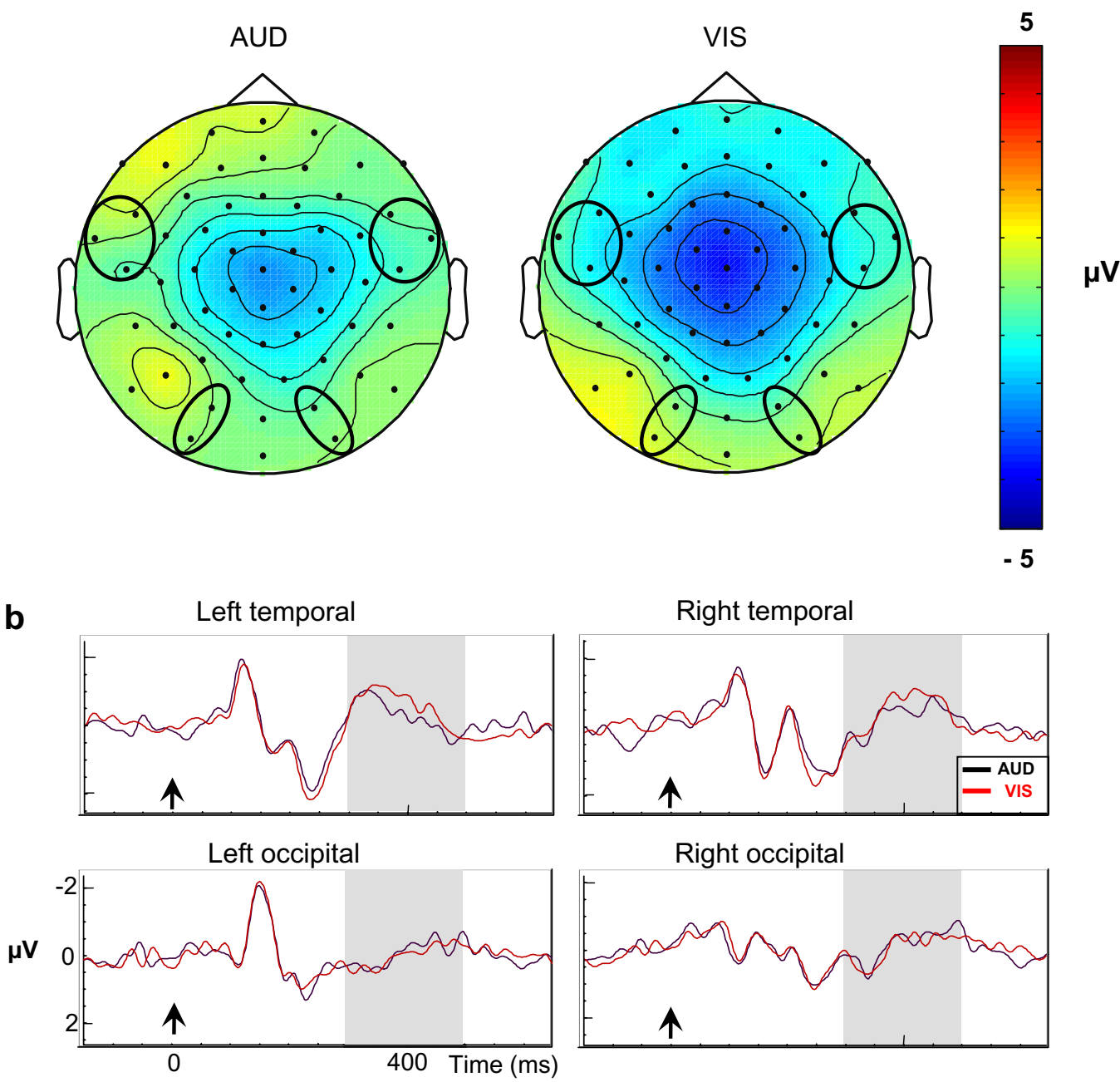

Fig. 2. (a) Scalp topography of the grand average $(N=16) \mathrm{N} 400$, as measured in the interval from $300 \mathrm{~ms}$ to 500 ms post-stimulus. Black ellipses indicate the channels used for each region of interest (ROI). Note the larger and slightly more frontal distribution of the N400 in the VIS condition. (b) ERP time courses for each of the four ROIs. The black arrows in each plot indicate word onset. The shaded areas indicate the time intervals used in the statistical analysis. Note the larger N400 for the VIS condition over both temporal ROIs.

between the two conditions, with the VIS words eliciting slightly more negativity at frontal channels than the AUD words (see also top graph in Fig. 1). The ANOVA indicates that the N400 is larger over temporal sites than over occipital sites (main effect of Area: $F_{1,15}=7.58$, $p=.007)$. Furthermore, the N400 was larger for VIS words than for AUD words at temporal ROIs, while no condition effect was found over occipital ROIs (interaction of Condition $*$ Area: $F_{1,15}=5.44, p=.017$; simple effect of Condition at temporal ROIS: $F_{1,15}=7.40, p=.016$; at occipital ROIs: $\left.F_{1,15}=0.11, p=.71\right)$.

\subsection{TFR data}

Fig. 3 presents the TFRs of the EEG power changes in the two conditions, over the predefined ROIs. Visual inspection of Fig. 3 suggests the presence of power increases in the theta band $(4-7 \mathrm{~Hz})$ and in the beta band
(13-18 Hz), and of power in- and decreases in the alpha band $(8-12 \mathrm{~Hz})$, all of which is in agreement with the existing literature. However, we found no clear indications of a power increase in the gamma frequency band. As a result, we defined four time-frequency components as follows: a theta component, from $4 \mathrm{~Hz}$ to $7 \mathrm{~Hz}$ and from $100 \mathrm{~ms}$ to $400 \mathrm{~ms}$ after word onset; an early alpha increase component, from $8 \mathrm{~Hz}$ to $12 \mathrm{~Hz}$ and from $50 \mathrm{~ms}$ to $150 \mathrm{~ms}$ after word onset; a late alpha decrease component, from $8 \mathrm{~Hz}$ to $12 \mathrm{~Hz}$ and from $300 \mathrm{~ms}$ to $700 \mathrm{~ms}$ after word onset; and a beta component, from $13 \mathrm{~Hz}$ to $8 \mathrm{~Hz}$ and from $75 \mathrm{~ms}$ to $175 \mathrm{~ms}$ after word onset.

\subsection{Power changes across all channels}

Before presenting the ROI-based, hypothesis-testing analysis of the four time-frequency components identified above, we first tested whether there were any overall differ- 


\section{Time-frequency representations}


Right occipital

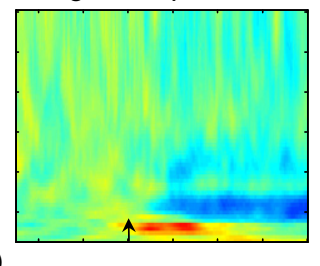

Fig. 3. Time-frequency representations of the grand average $(N=16)$ EEG power changes in both conditions for each of the four ROIs. The ROIs are indicated by ellipses in the head plot. Note the presence of a theta power increase (4-7 Hz), an alpha power (8-12 Hz) in- and subsequent decrease, and a beta power increase $(13-18 \mathrm{~Hz})$ in both conditions.

ences in power change between the AUD and VIS conditions in these time-frequency components across the entire scalp. We therefore averaged the power changes for all electrodes in the four time-frequency windows identified, separately for the two conditions. These data were subjected to a one-way ANOVA for repeated measures with the factor Condition (AUD, VIS). The results of these analyses show that for none of the four time-frequency components (theta, alpha increase, alpha decrease, beta increase) there were overall differences in power change between the two conditions across all channels (theta: $F_{1,15}=2.11, p<.167$; alpha increase: $F_{1,15}=0.380, p<.537$; alpha decrease: $F_{1,15}=0.764, p<.396$; beta increase: $F_{1,15}=2.13, p<.165$ ). Therefore, any topographical differences in power between the conditions in the ROI-based analysis cannot be attributed to overall differences in power across the entire scalp.

\subsection{Theta power increases}

Overall, there was a highly significant theta power increase $(21 \%$ increase on average) over the predefined ROIs $\left(F_{1,15}=65.22, p<.0001\right)$. Scalp topographies of the theta component are shown in Fig. 4a for both conditions. A left occipital maximum can be identified in both cases, with an additional peak at right occipito-parietal leads. This is supported by a main effect of Area $\left(F_{1,15}=3.65\right.$, $p=.038)$, and an interaction of Area * Hemisphere $\left(F_{1,15}=5.96, p=.014\right)$.

Interestingly, the scalp topography of the theta power increases over the a-priori defined ROIs show a consistent dependency on the type of stimulus (Fig. 4b), as indicated by an interaction of Condition * Area $\left(F_{1,15}=3.44\right.$, $p=.042$ ). This effect was however limited to the left-hemisphere ROIs (Condition* Area $*$ Hemisphere $\left(F_{1,15}=\right.$ 9.97, $p=0.003$; simple effect of Condition * Area in the left hemisphere: $F_{1,15}=11.20, p=.002$; simple effect of Condition $*$ Area in the right hemisphere: $F_{1,15}=0.566$, $p=.232$ ). In the left hemisphere, theta power increases were larger over the temporal ROI following AUD stimuli $\left(F_{1,15}=8.17, p=.006\right)$, and larger over the occipital ROI following VIS stimuli $\left(F_{1,15}=2.34, p=.07\right)$, although the latter effect is only marginally significant. This double dissociation, which confirms our hypothesis, can be observed in the two left-hand plots of Fig. 4b.

\subsection{Alpha power increases}

In the early time interval of $50-150 \mathrm{~ms}$ after stimulus onset an increase in alpha power was observed (Fig. 5). Overall, this power increase did not differ significantly from zero in the predefined ROIs (4\% power increase on average, $\left.F_{1,15}=0.97, p=.341\right)$. However, given the differences in scalp topography that can be observed in Fig. 5, this effect was subjected to the same statistical analysis as the other power changes. Scalp topographies of the alpha power increase show a parietal maximum in both conditions, but was more extended towards left occipital and right temporal areas in the VIS compared to the AUD condition (Fig. 5a). This was supported by the results of the statistical analysis: A significant interaction of Condition $*$ Area $*$ Hemisphere $\left(F_{1,15}=8.26, p=.006\right)$ 
a Theta $(4-7 \mathrm{~Hz})$ power changes
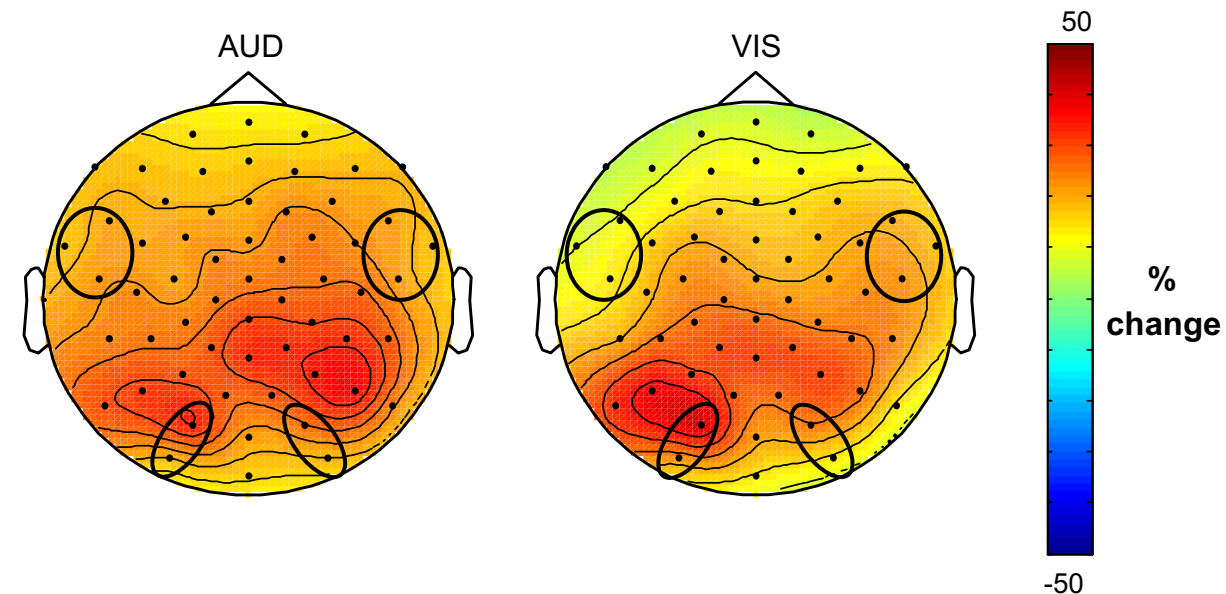

b
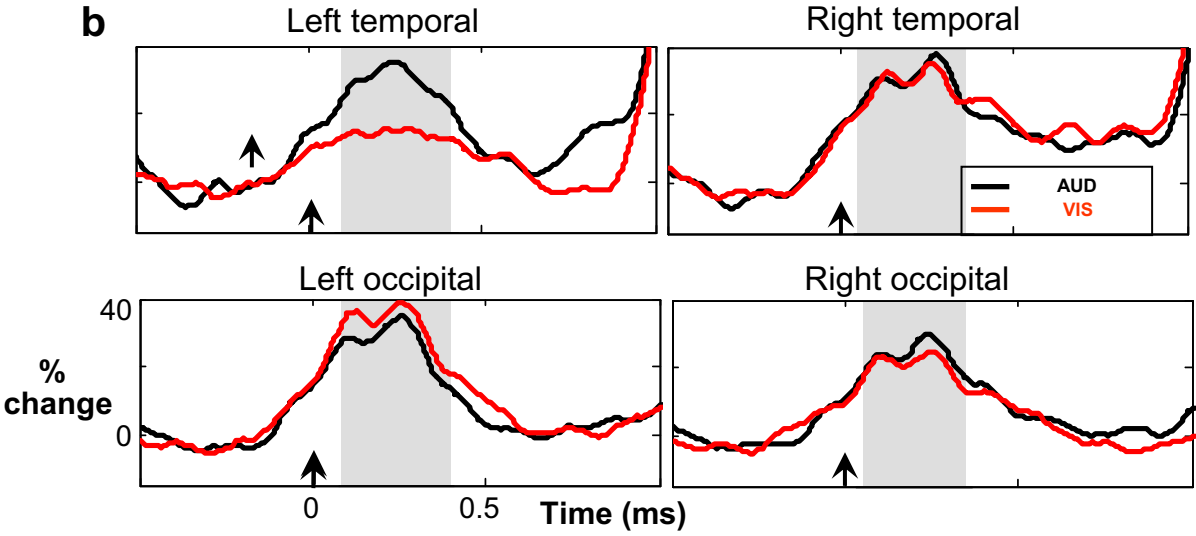

Fig. 4. (a) Scalp topography of the grand average $(N=16)$ theta power increase, from $4 \mathrm{~Hz}$ to $7 \mathrm{~Hz}$ and from $100 \mathrm{~ms}$ to $400 \mathrm{~ms}$ after word onset, for both conditions separately. Black ellipses indicate the channels used for each region of interest (ROI). Both conditions show a left occipital peak in theta power increase. (b) Temporal evolution of theta power change in each of the four ROIs. The black arrows in each plot indicate word onset. The shaded areas indicate the time intervals used in the statistical analysis. Note the opposite effects in the two left-hemisphere ROIs.

indicated that in the AUD condition, power increases were comparable across the four ROIs (Area * Hemisphere at AUD: $\left.F_{1,15}=1.44, p=.124\right)$. In contrast, in the VIS condition, power increases were larger over the right $(13 \%$ increase) than the left temporal ROI ( $3 \%$ decrease), and larger over the left $(15 \%$ increase) than the right $(2 \%$ decrease) occipital ROI (Area * Hemisphere at VIS: $F_{1,15}=9.92, \quad p=.003$; Hemisphere at VIS Temporal: $F_{1,15}=7.39, \quad p=.008$; Hemisphere at VIS Occipital: $\left.F_{1,15}=7.72, p=.007\right)$.

\subsection{Alpha power decreases}

There was an overall alpha power decrease over the predefined ROIs $(17 \%$ power decrease on average, $\left.F_{1,15}=5.26, p=.019\right)$. Scalp topographies of the alpha decrease (Fig. 5b) show a right occipito-temporal maximum in both conditions. The statistical analysis revealed a significant interaction of Condition with Area $\left(F_{1,15}=3.54, p=.04\right)$, suggesting a differential distribution of alpha power changes for the AUD and the VIS conditions. However, the underlying simple effects of Area in the AUD and VIS conditions were far from reaching significance (both $p$-values $>.55$ ), which makes the interaction uninterpretable. There were no other significant effects in this analysis.

\subsection{Beta power increases}

Overall, there was a significant beta power increase $(6 \%$ increase on average) over the predefined ROIs $\left(F_{1,15}=4.50, p=.025\right)$. Scalp topographies of the beta component (Fig. 6a) show a midcentral maximum for both conditions. The statistical analysis of this component reveals significant interactions of Area $*$ Hemisphere $\left(F_{1,15}=3.48, p=.041\right)$, and of Condition $*$ Area $*$ Hemisphere $\left(F_{1,15}=4.21, p=.029\right)$. Both interactions point to the fact that at the right occipital ROI, the beta power increase is larger following AUD stimuli than following VIS stimuli $\left(F_{1,15}=3.60, p=.034\right)$. 
a

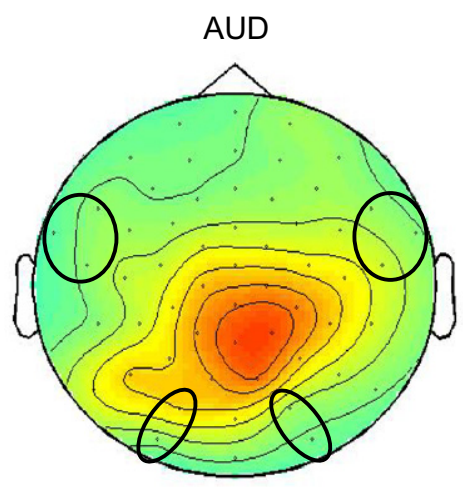

b

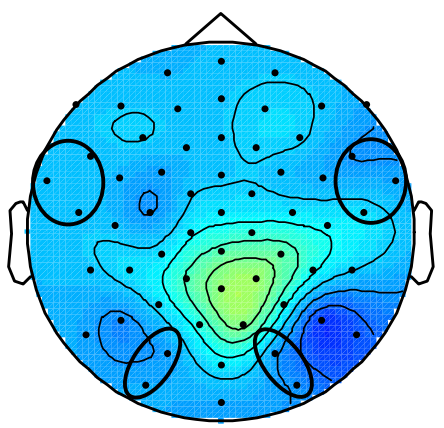

C

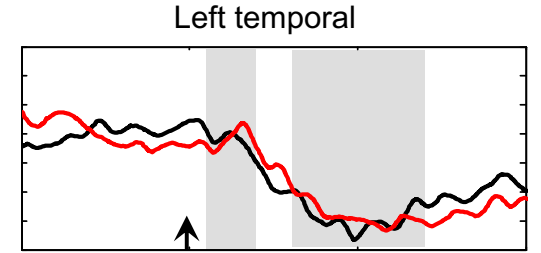

Left occipital



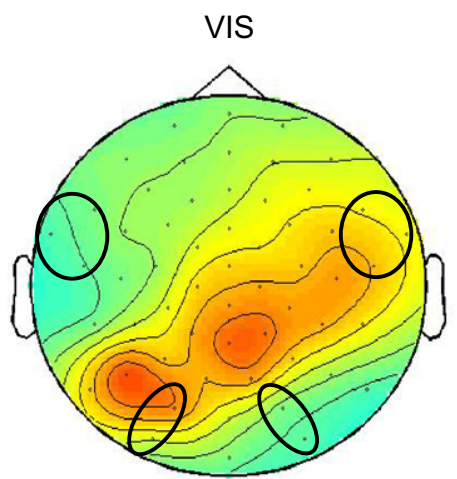

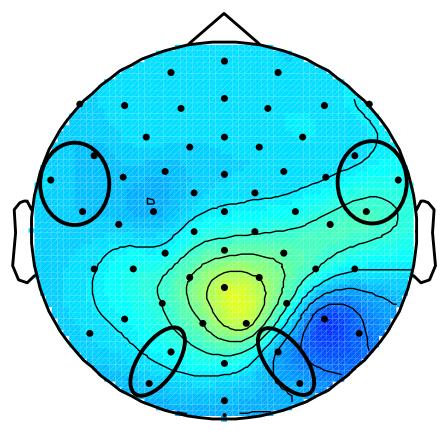

Right temporal

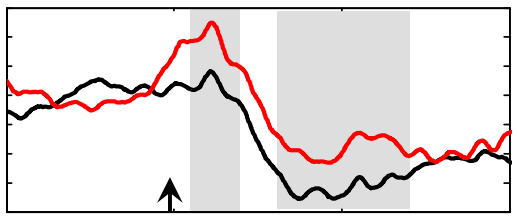

Right occipital

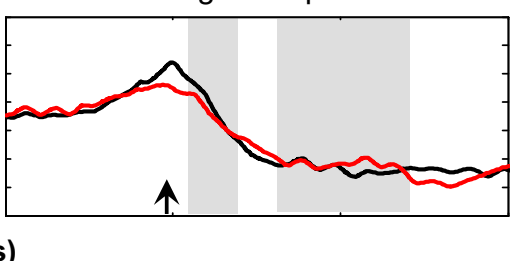

Fig. 5. (a) Scalp topography of the grand average alpha power increase, from $8 \mathrm{~Hz}$ to $12 \mathrm{~Hz}$ and from $50 \mathrm{~ms}$ to $150 \mathrm{~ms}$ after stimulus onset, for both conditions separately. Black ellipses indicate the channels used for each ROI. (b) Same as in (a), but now for the power decrease in a time window of 300 700 ms poststimulus. (c) Temporal evolution of alpha power change in each of the four ROIs. The black arrows in each plot indicate word onset. The shaded areas indicate the time intervals used in the statistical analysis.

\subsection{Time-frequency analysis of the ERP data}

The results of the time-frequency analysis of the timelocked averages are presented in Fig. 7. In the time-frequency plane, the ERP shows up as a power increase ranging roughly from $3 \mathrm{~Hz}$ to $12 \mathrm{~Hz}$ in the first $500 \mathrm{~ms}$ after stimulus onset. Note that, as baseline power in ERP data is generally very low due to the averaging procedure, the percent power change from baseline is much larger compared to the single-trial analyses.
The statistical analysis of the ERP theta power (see Fig. 7b, left-hand panel) does not yield any significant effects. The ANOVA on the ERP alpha power (see Fig. 7b, right-hand panel) shows that ERP alpha power is differently lateralized for the two conditions (interaction of Condition with Hemisphere: $F_{1,15}=4.70, p=.024$ ): In the AUD condition there is a right-hemisphere dominance $\left(F_{1,15}=5.28, p=.018\right)$, while in the VIS condition the ERP alpha power does not differ between hemispheres $\left(\left(F_{1,15}=1.13, p=.16\right)\right.$. There are no effects involving both 
a

Beta $(13-18 \mathrm{~Hz})$ power changes
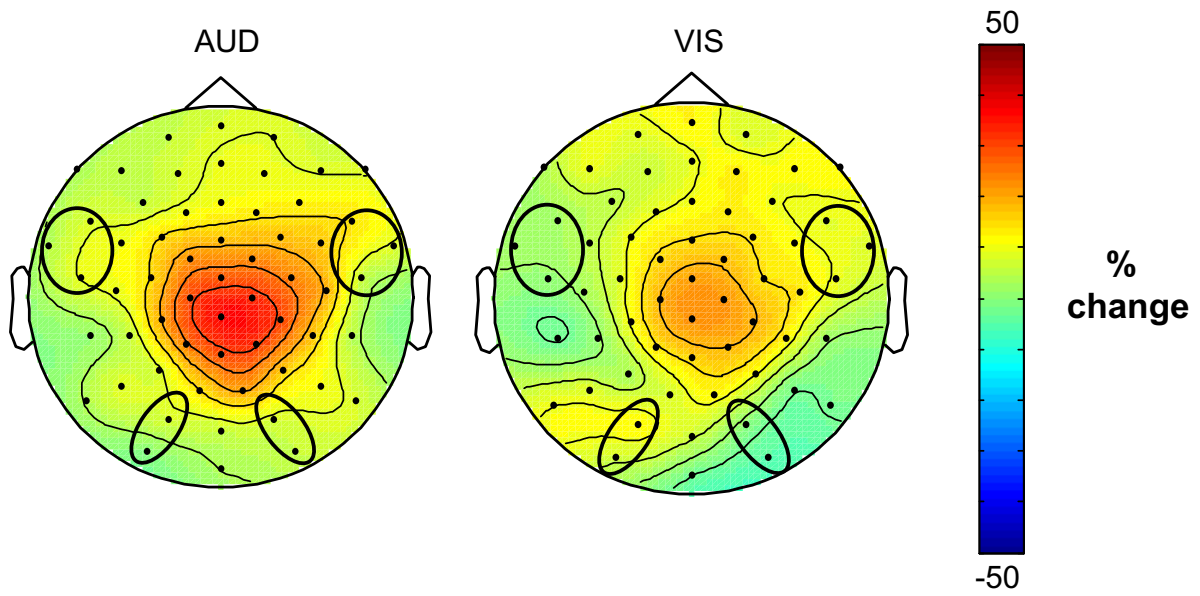

b

Left temporal

Right temporal
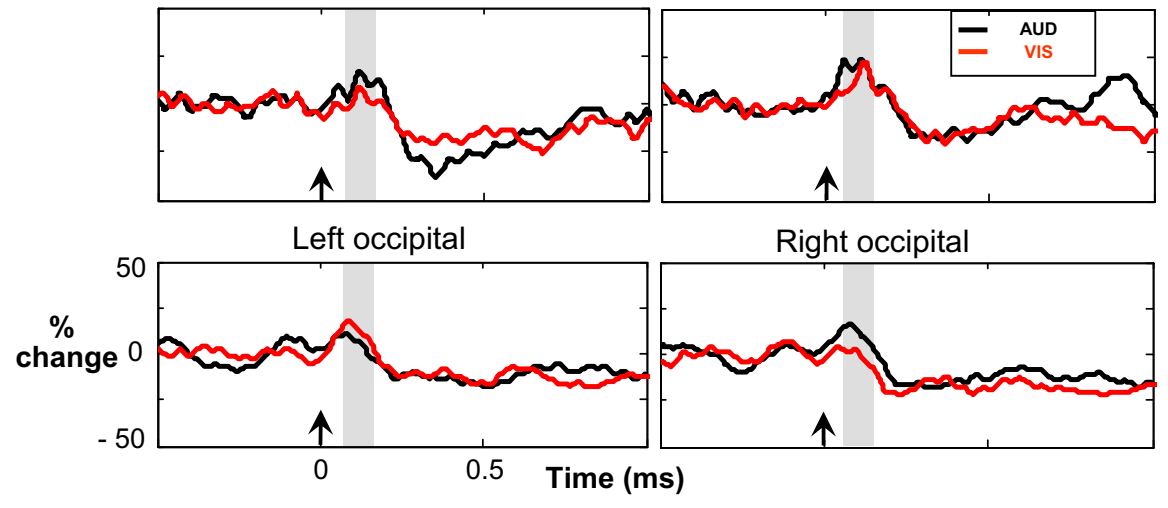

Fig. 6. (a) Scalp topography of the grand average beta power increase, from $13 \mathrm{~Hz}$ to $18 \mathrm{~Hz}$ and from $75 \mathrm{~ms}$ to $175 \mathrm{~ms}$ after word onset, for both conditions separately. Black ellipses indicate the channels used for each ROI. (b) Temporal evolution of beta power change in each of the four ROIs. The black arrows in each plot indicate word onset. The shaded areas indicate the time intervals used in the statistical analysis.

the factor Condition and the factor Area (despite the seemingly large condition difference over the left occipital ROI in the right-hand panel of Fig. 7b).

\section{Discussion}

We investigated event-related potentials (ERPs) and oscillatory brain dynamics of the EEG while subjects performed a visual lexical decision task. In agreement with our hypotheses, we found power increases in the theta band $(4-7 \mathrm{~Hz})$ and in the lower beta range $(13-18 \mathrm{~Hz})$. We also found the expected power decrease in the alpha $(8-12 \mathrm{~Hz})$ frequency range. However, in contrast to previous studies no evidence was found for power in- or decreases in the gamma frequency band.

The most striking finding of the present study is that the scalp topography of theta power changes was systematically influenced by the semantic properties of words. Words with auditory semantic properties (AUD; referring to sounds) elicited a larger theta power increase in electrodes overlying the left temporal cortex than words with visual semantic properties (VIS). This pattern was reversed in electrodes over the occipital cortex, with VIS words eliciting a larger theta power increase than AUD words (although the latter affect was only marginally significant).

While the theta band effects were in agreement with our hypothesis, in the alpha and gamma frequency bands no evidence was found for topographical differences between the two conditions. Note however, that the present, scalp-EEG-based study is not optimal for addressing power changes in the high end of the gamma frequency range $(70 \mathrm{~Hz}$ and above. Therefore, the conclusion that no topographical differences in gamma power were found between conditions is necessarily limited to the lower end of the gamma frequency range (roughly $30-60 \mathrm{~Hz}$ ). For the beta frequency band, we did find a topographical difference between the two conditions (a larger power increase over the right occipital area for AUD words). However, this effect was restricted to the right hemisphere, and no condition effects were observed over temporal regions. Therefore, it is difficult to relate the observed condition difference to our hypothesis on semantic representations. 
Time-frequency analysis of ERP data

a

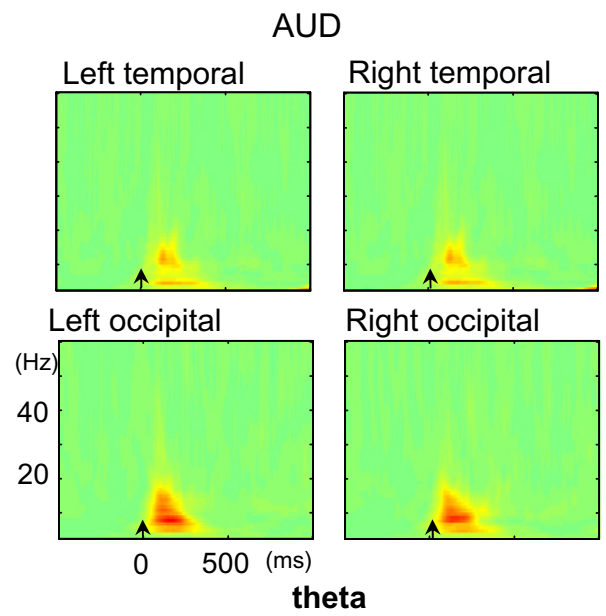

b
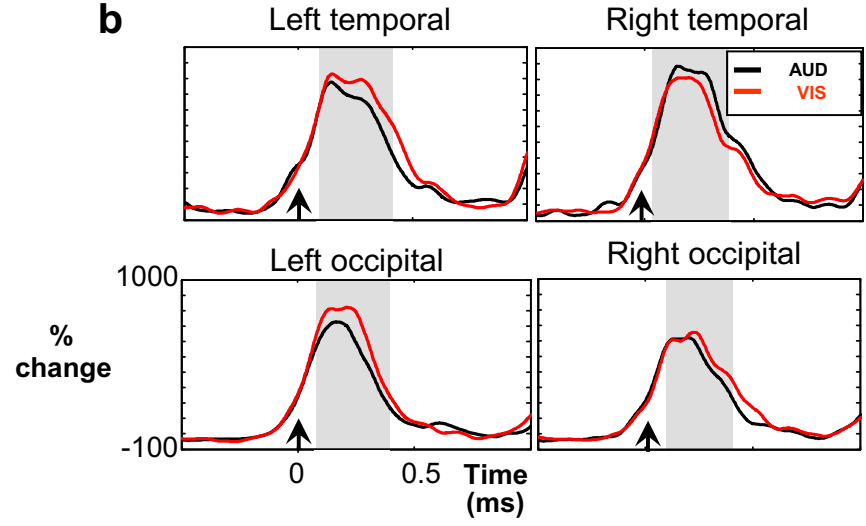
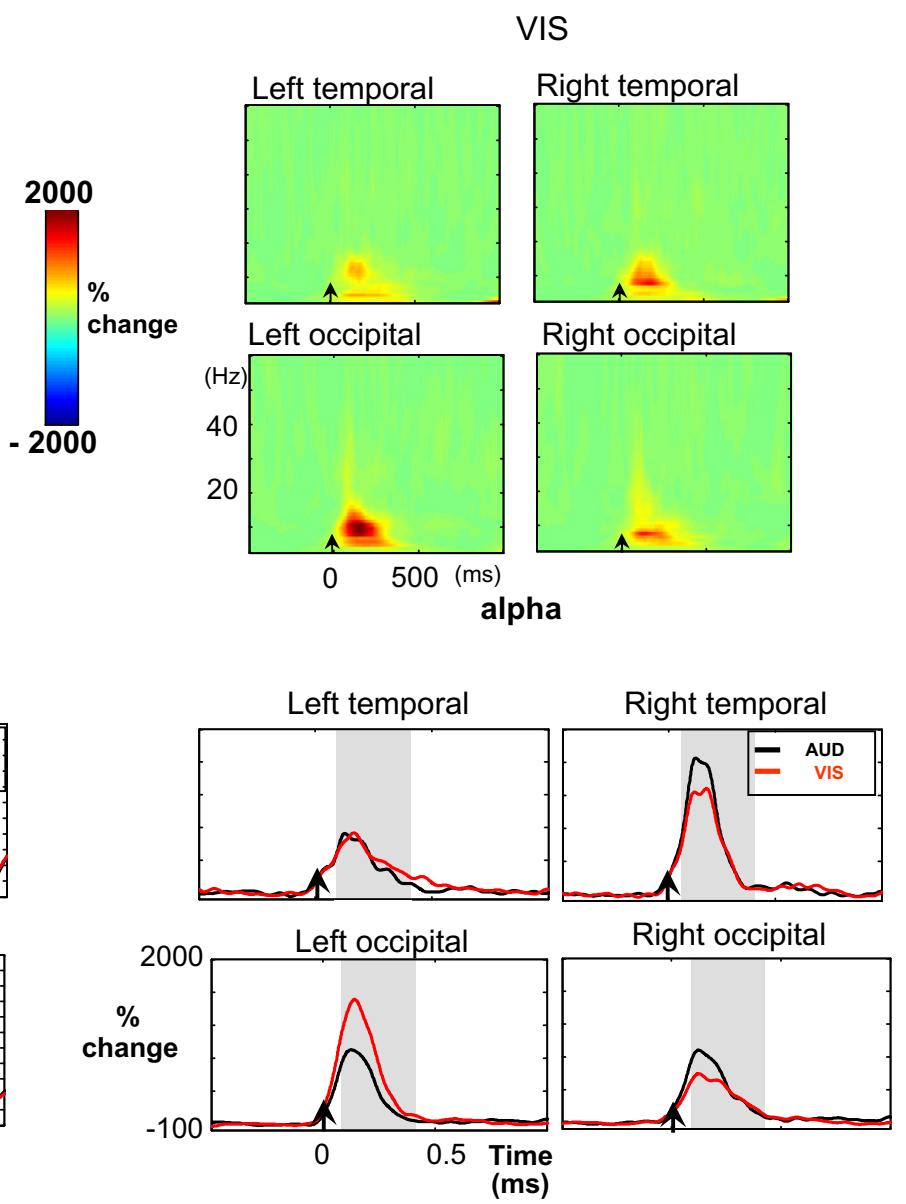

Fig. 7. (a) Grand-average time-frequency representations of the ERP data, for each of the ROIs. Note that the ERP shows up in the time-frequency plane as a power increase between roughly $3-12 \mathrm{~Hz}$. (b) Temporal evolution of theta $(4-7 \mathrm{~Hz}$, left-hand panel) and alpha (8-12 Hz), right-hand panel) power changes in each of the four ROIs. The black arrows in each plot indicate word onset. The shaded areas indicate the time intervals used in the statistical analysis.

Thus, as far as the oscillatory dynamics are concerned, the only effect that systematically varies with semantic properties is observed in the theta frequency band.

For the N400, we observed larger amplitudes over temporal cortex following VIS stimuli compared to AUD stimuli. However, judging from Figs. 1 and 2 this was caused by larger overall N400 amplitude for the VIS stimuli, especially at frontal electrode sites. Since larger N400 amplitudes are sometimes related to a lexical search (c.f. the larger N400 amplitude commonly observed after pseudowords compared to words), this effect could be related to a more extensive lexical search process following VIS stimuli compared to AUD stimuli, despite the close matching of lexical parameters between the two conditions. Alternatively, and more probably, in our opinion, N400 differences might have been caused by differences in imageability between the conditions: It has been reported repeatedly that high imageable words elicit a frontally larger N400 than low imageable words (Holcomb, Kounios, Anderson, \& West, 1999; Kounios \& Holcomb, 1994; Swaab, Baynes, $\&$ Knight, 2002). Since VIS words likely have higher image- ability than AUD words, this would explain our observed N400 differences between AUD and VIS conditions. In any case, the N400 data cannot be taken to support the hypotheses.

The lack of a clear double dissociation in the ERP data indicates that time-frequency analysis of oscillatory components (here in the theta-band in particular) has the potential to yield new information compared to the more traditional ERP analyses. It also shows that oscillatory responses are more than just time-frequency representations of the ERP. The latter is explicitly supported by the fact that the time-frequency analysis of the ERP data shows no effects of semantic properties in the theta frequency range (nor in the alpha frequency range, for that matter). This excludes the possibility that the semantic property-specific theta power increases we have found are brought about by differences in evoked activity. Instead, the observed theta response is exclusively an induced (non-phase-locked) phenomenon (see e.g., Bastiaansen \& Hagoort, 2003; Makeig et al., 2002 for discussions of these concepts). This suggest that the observed theta power 
increase is a modulation of an oscillatory process, even though it is relatively short-lived (it lasts about $300 \mathrm{~ms}$, approximately $1-2$ theta cycles).

As both sets of words were presented visually, and were closely matched for word length and for word frequency, the double dissociation in the scalp topography of the oscillatory theta responses is produced exclusively by the different semantic properties of the lexical items. As such, our data are in agreement with the hypothesis that spatially distributed functional networks form the basis of semantic representations, and that the topographies of these networks reflect the semantic properties of individual items (Martin \& Chao, 2001; Pulvermueller, 1999, 2001). In relation to the latter claim, however, we wish to emphasize that our data do not allow us to draw firm conclusions with respect to the precise topographical distribution of the semantic networks in the brain, because there is not a one-to-one correspondence between our ROIs (which are based on scalp-recorded EEG) and the underlying auditory and visual cortices.

In addition to the above, our data add a new and distinctive element to our understanding of the retrieval/activation of lexical semantics, which is that we seem to have identified a dynamic neuronal mechanism that implements the activation/recruitment of such a network, namely synchronization of neuronal activity at theta frequencies. In contrast with the relatively static information that fMRI studies yield, our current approach therefore informs us (at least to some extent) not only on the fact that such networks become activated, but also on how they become activated.

This hypothesis is supported by our earlier observations (see Bastiaansen \& Hagoort, 2006 for a review) that theta power increases are related to lexico-semantic retrieval operations (Bastiaansen, Van der Linden, ter Keurs, Dijkstra, \& Hagoort, 2005), and that theta power changes are sensitive to semantic violations occurring in a sentence context (Hagoort et al., 2004; Hald, Bastiaansen, \& Hagoort, 2006). In addition, indirect support for the hypothesis that theta power changes are involved in the activation of semantically specific functional networks stems from a number of studies which have demonstrated a more general relation between theta power changes and memory encoding/retrieval operations. For instance, theta oscillations have been found to increase in power during encoding and retrieval of episodic information from long-term memory (e.g., Burgess \& Ali, 2002; Fell et al., 2001; Klimesch, 1999; Klimesch, Doppelmayr, Russeger, \& Pachinger, 1996; Klimesch, Doppelmayr, Schimke, \& Ripper, 1997c), and seem to be more strongly involved in retrieval than in encoding (Klimesch et al., 2001). Theta power changes have also been related to working memory processes (e.g., Bastiaansen, Posthuma, Groot, \& de Geus, 2002a; Jensen \& Tesche, 2002; Kahana, Sekuler, Caplan, Kirschen, \& Madsen, 1999; Tesche \& Karhu, 2000). Note, however, that we do not want to claim that theta-band neuronal synchronization is the sole mechanism by which lex- ical retrieval is realized. Although this mechanism may be instrumental in recruiting relevant cortical areas, we do not exclude the possibility that other dynamic processes are involved in lexical retrieval as well.

Finally, it should be emphasized that although the semantic properties of lexical items influence the topography of theta power changes, they certainly do not determine this topography. In fact, for none of the time-frequency components we analysed did we find topographies that were maximal over the temporal or occipital cortex. For the theta frequency range, power was maximal over left occipital areas for both conditions. This could potentially be related to activity in the fusiform gyrus (the so-called visual word form area), as we have argued in some detail elsewhere (Bastiaansen et al., 2005), but firm evidence for this notion is lacking. The alpha increase is maximal over midparietal areas, a finding that might speculatively be related to attentional processes (see e.g., Babiloni et al., 2004; Dockree, Kelly, Foxe, Reilly, \& Robertson, 2007; Laufs et al., 2003). The alpha decrease is maximal over left and right occipital areas, an effect that has often been related to (domain-general) visual processing. The beta increase has its maximum at the vertex, and is the most difficult to interpret in terms of topographical distribution.

In conclusion, the semantic properties of visually presented words were found to partly determine the topography of EEG theta power increases in a lexical decision task. Our data are thus in agreement with the view that semantic representations are stored in functional networks with a topography that corresponds to the semantic properties of the stored items. In addition, our data suggest that oscillatory brain dynamics in the theta frequency range are involved in the retrieval of lexical-semantic information.

\section{References}

Baayen, R. H., Piepenbrock, R., \& van Rijn, H. (1993). The CELEX lexical database (CD-ROM). Philadelphia: University of Pennsylvania.

Babiloni, C., Miniussi, C., Babiloni, F., Carducci, F., Cincotti, F., Del Percio, C., et al. (2004). Sub-second "temporal attention" modulates alpha rhythms. A high-resolution EEG study. Brain Research. Cognitive Brain Research, 19(3), 259-268.

Bastiaansen, M. C. M., \& Hagoort, P. (2003). Event-induced theta responses as a window on the dynamics of memory. Cortex, 39(4-5), 967-992.

Bastiaansen, M. C. M., \& Hagoort, P. (2006). Oscillatory brain dynamics during language comprehension. In W. Klimesch \& C. Neuper (Eds.). Event-related dynamics of brain oscillations (159, pp. 182-196). Amsterdam: Elsevier.

Bastiaansen, M. C. M., Posthuma, D., Groot, P. F., \& de Geus, E. J. (2002a). Event-related alpha and theta responses in a visuo-spatial working memory task. Clinical Neurophysiology, 113(12), 1882-1893.

Bastiaansen, M. C. M., van Berkum, J. J., \& Hagoort, P. (2002b). Eventrelated theta power increases in the human EEG during online sentence processing. Neuroscience Letters, 323(1), 13-16.

Bastiaansen, M. C. M., van Berkum, J. J., \& Hagoort, P. (2002c). Syntactic processing modulates the theta rhythm of the human EEG. Neuroimage, 17(3), 1479-1492.

Bastiaansen, M. C. M., Van der Linden, M., ter Keurs, M., Dijkstra, T., \& Hagoort, P. (2005). Theta responses are involved in lexico-semantic 
retrieval during language processing. Journal of Cognitive Neuroscience, 17(3), 530-541.

Burgess, A. P., \& Ali, L. (2002). Functional connectivity of gamma EEG activity is modulated at low frequency during conscious recollection. International Journal of Psychophysiology, 46(2), 91-100.

Damasio, A. R. (1990). Category-related recognition defects as a clue to the neural substrates of knowledge. Trends in Neurosciences, 13(3), 95-98.

Devlin, J. T., Russell, R. P., Davis, M. H., Price, C. J., Moss, H. E., Fadili, M. J., et al. (2002). Is there an anatomical basis for categoryspecificity? Semantic memory studies in PET and fMRI. Neuropsychologia, 40(1), 54-75.

Dockree, P. M., Kelly, S. P., Foxe, J. J., Reilly, R. B., \& Robertson, I. H. (2007). Optimal sustained attention is linked to the spectral content of background EEG activity: Greater ongoing tonic alpha (approximately $10 \mathrm{~Hz}$ ) power supports successful phasic goal activation. The European Journal of Neuroscience, 25(3), 900-907.

Farah, M. J., \& McClelland, J. L. (1991). A computational model of semantic memory impairment: Modality specificity and emergent category specificity. Journal of Experimental Psychology. General, 120(4), 339-357.

Fell, J., Klaver, P., Lehnertz, K., Grunwald, T., Schaller, C., Elger, C. E., et al. (2001). Human memory formation is accompanied by rhinalhippocampal coupling and decoupling. Nature Neuroscience, 4(12), 1259-1264.

Forde, E. M. E., \& Humphreys, G. W. (1999). Category-specific recognition impairments: A review of important case studies and influential theories. Aphasiology, 13(3), 169-193.

Hagoort, P., Hald, L., Bastiaansen, M., \& Petersson, K. M. (2004). Integration of word meaning and world knowledge in language comprehension. Science, 304(5669), 438-441.

Hald, L. A., Bastiaansen, M. C., \& Hagoort, P. (2006). EEG theta and gamma responses to semantic violations in online sentence processing. Brain and Language, 96(1), 90-105.

Hauk, O., Johnsrude, I., \& Pulvermueller, F. (2004). Somatotopic representation of action words in human motor and premotor cortex. Neuron, 41(2), 301-307.

Hauk, O., \& Pulvermueller, F. (2004). Neurophysiological distinction of action words in the fronto-central cortex. Human Brain Mapping, 21(3), 191-201.

Holcomb, P. J., Kounios, J., Anderson, J. E., \& West, W. C. (1999). Dualcoding, context-availability, and concreteness effects in sentence comprehension: An electrophysiological investigation. Journal of Experimental Psychology. Learning, Memory, and Cognition, 25(3), $721-742$.

Jensen, O., \& Tesche, C. D. (2002). Frontal theta activity in humans increases with memory load in a working memory task. The European Journal of Neuroscience, 15(8), 1395-1399.

Kahana, M. J., Sekuler, R., Caplan, J. B., Kirschen, M., \& Madsen, J. R. (1999). Human theta oscillations exhibit task dependence during virtual maze navigation. Nature, 399, 781-784.

Klimesch, W. (1999). EEG alpha and theta oscillations reflect cognitive and memory performance: A review and analysis. Brain Research Reviews, 29, 169-195.

Klimesch, W., Doppelmayr, M., Pachinger, T., \& Ripper, B. (1997a). Brain oscillations and human memory: EEG correlates in the upper alpha and theta band. Neuroscience Letters, 238(1-2), 9-12.

Klimesch, W., Doppelmayr, M., Pachinger, T., \& Russegger, H. (1997b). Event-related desynchronization in the alpha band and the processing of semantic information. Brain Research. Cognitive Brain Research, 6(2), 83-94.

Klimesch, W., Doppelmayr, M., Russeger, H., \& Pachinger, T. (1996). Theta band power in the human scalp EEG and the encoding of new information. Neuroreport, 7, 1235-1240.

Klimesch, W., Doppelmayr, M., Schimke, H., \& Ripper, B. (1997c). Theta synchronization in a memory task. Psychophysiology, 34, 169-176.

Klimesch, W., Doppelmayr, M., Stadler, W., Pollhuber, D., Sauseng, P., \& Rohm, D. (2001). Episodic retrieval is reflected by a process-specific increase in human electroencephalographic theta activity. Neuroscience Letters, 302, 49-52.

Kounios, J., \& Holcomb, P. J. (1994). Concreteness effects in semantic processing: ERP evidence supporting dual-coding theory. Journal of Experimental Psychology. Learning, Memory, and Cognition, 20(4), 804-823.

Laufs, H., Krakow, K., Sterzer, P., Eger, E., Beyerle, A., Salek-Haddadi, A., et al. (2003). Electroencephalographic signatures of attentional and cognitive default modes in spontaneous brain activity fluctuations at rest. Proceedings of the National Academy of Sciences of the United States of America, 100(19), 11053-11058.

Makeig, S., Westerfield, M., Jung, T. P., Enghoff, S., Townsend, J., Courchesne, E., et al. (2002). Dynamic brain sources of visual evoked responses. Science, 295(5555), 690-694.

Martin, A., \& Chao, L. L. (2001). Semantic memory and the brain: Structure and processes. Current Opinion in Neurobiology, 11(2), 194-201.

Martin, A., Haxby, J. V., Lalonde, F. M., Wiggs, C. L., \& Ungerleider, L. G. (1995). Discrete cortical regions associated with knowledge of color and knowledge of action. Science, 270(5233), 102-105.

Martin, A., Wiggs, C. L., Ungerleider, L. G., \& Haxby, J. V. (1996). Neural correlates of category-specific knowledge. Nature, 379(6566), 649-652.

Mitra, P. P., \& Pesaran, B. (1999). Analysis of dynamic brain imaging data. Biophysics Journal, 76(2), 691-708.

Noppeney, U., \& Price, C. J. (2002). Retrieval of visual, auditory, and abstract semantics. Neuroimage, 15(4), 917-926.

Pfurtscheller, G., \& Aranibar, A. (1977). Event-related cortical desynchronization detected by power measurements of scalp EEG. Electroencephalography and Clinical Neurophysiology, 42, 817-826.

Pfurtscheller, G., \& Aranibar, A. (1979). Evaluation of event-related desynchronization (ERD) preceding and following voluntary selfpaced movements. Electroencephalography and Clinical Neurophysiology, 46, 138-146.

Pfurtscheller, G., \& Berghold, A. (1989). Patterns of cortical activation during planning of voluntary movement. Electroencephalography and Clinical Neurophysiology, 72, 250-258.

Pfurtscheller, G., \& Lopes da Silva, F. H. E. (1999). Event-related desynchronization. Handbook of electroencephalography and clinical neurophysiology revised series (Vol. 6). Amsterdam: Elsevier.

Pulvermueller, F. (1999). Words in the brain's language. The Behavioral and Brain Sciences, 22, 253-336.

Pulvermueller, F. (2001). Brain reflections of words and their meaning. Trends Cognitive Sciences, 5(12), 517-524.

Pulvermueller, F., Eulitz, C., Pantev, C., Mohr, B., Feige, B., Lutzenberger, W., et al. (1996). High-frequency cortical responses reflect lexical processing: An MEG study. Electroencephalography and Clinical Neurophysiology, 98(1), 76-85.

Pulvermueller, F., Harle, M., \& Hummel, F. (2001). Walking or talking? Behavioral and neurophysiological correlates of action verb processing. Brain and Language, 78, 143-168.

Pulvermueller, F., Lutzenberger, W., \& Preissl, H. (1999). Nouns and verbs in the intact brain: Evidence from event-related potentials and high-frequency cortical responses. Cerebral Cortex, 9(5), 497-506.

Rohm, D., Klimesch, W., Haider, H., \& Doppelmayr, M. (2001). The role of theta and alpha oscillations for language comprehension in the human electroencephalogram. Neuroscience Letters, 310(2-3), 137-140.

Saffran, E. M., \& Sholl, A. (1999). Clues to the functional and neural architecture of word meaning. In C. Brown \& P. Hagoort (Eds.), The neurocognition of language (pp. 241-272). Oxford: Oxford University Press.

Sakowitz, O. W., Quian Quiroga, R., Schurmann, M., \& Basar, E. (2005). Spatio-temporal frequency characteristics of intersensory components in audiovisually evoked potentials. Brain Research. Cognitive Brain Research, 23(2-3), 316-326.

Singer, W. (1999). Neuronal synchrony: A versatile code for the definition of relations? Neuron, 24(1), 49-65, 111-125. 
Swaab, T. Y., Baynes, K., \& Knight, R. T. (2002). Separable effects of priming and imageability on word processing: An ERP study. Brain Research. Cognitive Brain Research, 15(1), 99-103.

Tallon-Baudry, C., Bertrand, O., Delpuech, C., \& Pernier, J. (1996). Stimulus specificity of phase-locked and non-phase-locked $40 \mathrm{~Hz}$ visual responses in human. Journal of Neuroscience, 16(13), 4240-4249.

Tesche, C. D., \& Karhu, J. (2000). Theta oscillations index human hippocampal activation during a working memory task. Proceedings of the National Academy of Sciences of the United States of America, 97, 919-924.

Tyler, L. K., \& Moss, H. E. (2001). Towards a distributed account of conceptual knowledge. Trends in Cognitive Science, 5(6), 244-252.

Tyler, L. K., Moss, H. E., Durrant-Peatfield, M. R., \& Levy, J. P. (2000). Conceptual structure and the structure of concepts: A distributed account of category-specific deficits. Brain and Language, 75(2), 195-231.

Varela, F., Lachaux, J. P., Rodriguez, E., \& Martinerie, J. (2001). The brainweb: Phase synchronization and large-scale integration. Natural Reviews. Neuroscience, 2(4), 229-239.

Vitali, P., Abutalebi, J., Tettamanti, M., Rowe, J., Scifo, P., Fazio, F., et al. (2005). Generating animal and tool names: An fMRI study of effective connectivity. Brain and Language, 93(1), 32-45.

Warrington, E. K., \& McCarthy, R. A. (1987). Categories of knowledge. Further fractionations and an attempted integration. Brain, 110(Pt. 5), 1273-1296.

Warrington, E. K., \& Shallice, T. (1984). Category specific semantic impairments. Brain, 107(Pt. 3), 829-854.

Weiss, S., \& Mueller, H. M. (2003). The contribution of EEG coherence to the investigation of language. Brain and Language, 85(2), 325-343. 